Artículo científico

Volumen 31(3):547-565. Septiembre-diciembre, 2020

e-ISSN 2215-3608, doi:10.15517/am.v31i3.37334

http://www.revistas.ucr.ac.cr/index.php/agromeso

\title{
Efecto del fertilizante orgánico y mineral en rendimiento de caña panelera en Nariño, Colombia ${ }^{1}$
}

\section{Effect of the organic and mineral fertilizer on the performance of sugarcane yield in Nariño, Colombia}

\author{
Belisario Volverás-Mambuscay², Carlos Felipe González-Chavarro ${ }^{3}$, Bellanid Huertas ${ }^{4}$,Eugenio Kopp-Sanabria ${ }^{3}$, \\ Julio Ramírez-Durán ${ }^{5}$
}

1 Recepción: 7 de octubre, 2019. Aceptación: 4 de marzo, 2020. Este trabajo formó parte del proyecto ajuste de prácticas culturales para un adecuado manejo agronómico del cultivo de caña de azúcar (Saccharum officinarum L.) en cinco regiones paneleras del país llevado a cabo en la Corporación Colombiana de Investigación Agropecuaria - AGROSAVIA, Colombia.

2 Corporación Colombiana de Investigación Agropecuaria - AGROSAVIA, Centro de Investigación Obonuco - km 5 vía Pasto, Nariño, Colombia, código postal 684511. bvolveras@agrosavia.co (https://orcid.org/0000-0002-6571-670X).

3 Corporación Colombiana de Investigación Agropecuaria - AGROSAVIA, Centro de Investigación Tibaitatá sede Cimpa - km 2 vía antigua a Cite - Barbosa, Santander, Colombia, código postal 684511, Colombia. cfgonzalez@agrosavia.co (autor para correspondencia, https://orcid. org/0000-0002-1720-5067), ekopp@agrosavia.co (https://orcid.org/0000-0003-4099-5236).

4 Corporación Colombiana de Investigación Agropecuaria - AGROSAVIA, Centro de Investigación Tibaitatá - km 14 vía Mosquera - Bogotá, Cundinamarca, Colombia, código postal 684511. bhuertas@agrosavia.co (https://orcid.org/0000-0002-1034-4072).

5 Corporación Colombiana de Investigación Agropecuaria - AGROSAVIA, Centro de Investigación Tibaitatá - km 14 Mosquera - Bogotá, Cundinamarca, Colombia, código postal 250047. jramirezd@agrosavia.co (https://orcid.org/0000-0002-3385-5748).

\section{Resumen}

Introducción. Colombia es el segundo productor de panela o azúcar no centrifugada (ANC) en el mundo, después de la India. En 2016, el departamento de Nariño tuvo un área en caña de azúcar (Saccharum officinarum L.) para panela o ANC cosechada de 10533 ha y un rendimiento promedio de 7,67 t ha ${ }^{-1}$ para un 6,35\% de la producción total de panela o ANC en el país. Objetivo. Evaluar el uso de fertilizante orgánico y mineral sobre el rendimiento en caña de azúcar para producción de panela o ANC en su primer ciclo de producción. Materiales y métodos. El estudio se efectuó entre los meses de noviembre de 2008 a marzo de 2010 en la vereda Villa Inés, Consacá, Nariño; se empleó un diseño de bloques completos al azar, considerando sistemas de siembra estaca continúa (SEC) y espaciado (SE) niveles de N-P-K ajustados según análisis de suelo, con fuentes químicas (FQ) y orgánicas (FO). Se evaluó rendimiento en caña y panela o ANC y azúcares reductores y fosfatos. Resultados. Los tratamientos (T3) SEC-FQ con 168,20 TCH, (T1) SEC-FA con 167,07 TCH y (T5) SE-AO con 167 TCH presentaron el mayor rendimiento de caña con respecto a los demás tratamientos, y fueron significativamente diferentes al tratamiento del testigo (T9). En panela o ANC, el mejor tratamiento fue (T6) SE-FQ (12,1\% de conversión y 18,78 t ha-1). El mayor valor de azúcares reductores lo mostró (T8) SE-FA con 13,2 \%, mientras en fosfatos fue (T9) SEC y SE-SF (753 ppm), y SEC-AO (706 ppm). Con $1500 \mathrm{~kg} \mathrm{ha}^{-1}$ de abono orgánico comercial se obtuvo rendimientos de caña por arriba de $100 \mathrm{t} \mathrm{ha}^{-1}$ y rendimientos de panela o ANC de 15 y $17 \mathrm{t} \mathrm{ha}^{-1}$ en SEC y SE respectivamente, similares a los alcanzados con fertilización química. En la fertilización química, con la mezcla de fuentes se redujo el uso de nitrógeno en 42 $\%$ y de fósforo en $18 \%$, con rendimiento de caña $44 \%$ superior al promedio regional y de panela $20 \%$ superior al 
testigo. Conclusión. Fue posible reducir y suplir algunos nutrientes de síntesis química sin afectar el rendimiento de caña y panela o ANC.

Palabras clave: azúcar no centrifugada, complejos organominerales, productividad, nutrición de las plantas .

\begin{abstract}
Introduction. Colombia is the second producer of panela or non-centrifugal sugar (NCS) in the world, after India. In 2016, the department of Nariño had an area in sugarcane (Saccharum officinarum L.) for harvested sugarcane or NCS of 10533 ha and an average yield of $7.67 \mathrm{t} \mathrm{ha}^{-1}$ for $6.35 \%$ of total production of panela or NCS in the country. Objective. To evaluate the use of organic and mineral fertilizer on the sugarcane yield for panela or NCS production in its first production cycle. Materials and methods. The study was carried out between the months of November 2008 to March 2010 in the village of Villa Ines, Consaca, Nariño; a randomized complete block design was used, considering continuous sowing systems (SEC) and spacing (SE) ajusted NPK levels according to soil analysis, with chemical (FQ) and organic (FO) sources. The yield was evaluated in cane and panela or NCS, and reducing sugars and phosphates. Results. The treatments (T3) SEC-FQ with 168.20 TCH, (T1) SEC-FA with 167.07 TCH and (T5) SE-AO with $167 \mathrm{TCH}$ showed the highest cane yield compared to the other treatments, and where significantly different from the control treatment (T9). In panela or NCS, the best treatment was (T6) SE-FQ (12.1\% conversion and $\left.18.78 \mathrm{tha}^{-1}\right)$. The highest value of reducing sugars was (T8) SE-FA with $13.2 \%$ while in phosphates it was (T9) SEC and SE-SF (753 ppm), and SEC-AO (706 ppm). With 1,500 kg ha ${ }^{-1}$ of commercial organic fertilizer, cane yields above $100 \mathrm{t}^{-1}$ and panela yields or NCSof 15 and $17 \mathrm{t} \mathrm{ha}^{-1}$ in SEC and SE, respectively, were obtained, similar to those achieved with chemical fertilization. In chemical fertilization, with the mixture of sources the use of nitrogen was reduced by $42 \%$ and phosphorus by $18 \%$, with cane yields $44 \%$ higher than the regional average and panela yields $20 \%$ higher than the control. Conclusion. It was possible to reduce and supply some nutrients of chemically synthesized nutrients without affecting the sugarcane and panela or NCS yields.
\end{abstract}

Keywords: non-centrifugal sugar, organo-mineral complexes, productivity, plant nutrition.

\title{
Introducción
}

Colombia es el segundo productor de panela o azúcar no centrifugada (ANC) en el mundo, después de India, y también es el país líder en consumo per cápita, con un promedio de 24,7 $\mathrm{kg}$ año $^{-1}$ (Gutiérrez-Mosquera et al., 2018). La agroindustria panelera es de gran importancia para la economía nacional, ocupando la segunda línea en la oferta de empleo para el sector agrícola, adicional a esto la agroindustria panelera de manejo familiar en Colombia, se mantiene como consecuencia del alto valor nutritivo del producto, su adaptación a diversas condiciones de suelos y clima, y bajo ingreso de insumos agrícolas.

En 2016, el departamento de Nariño tuvo un área en caña de azúcar (Saccharum officinarum L.) para panela o ANC cosechada de 10533 ha y un rendimiento promedio de 7,67 t ha ${ }^{-1}$ contribuyendo con 6,35\% de la producción total de panela o ANC en el país (Agronet, 2016). En esta localidad se estima que cada hectárea cultivada requiere de aproximadamente 128 jornales al año y cada trapiche con una capacidad de $100 \mathrm{~kg}$ de panela o ANC por hora, genera catorce puestos de trabajo y favorece otros sectores productivos por su alta demanda en la industria de fertilizantes, empaques, transporte, metalmecánica y comercialización (Luna, 1992; Campuzano y Bolaños, 2003; DANE, 2017). 
La agroindustria panelera en Colombia, a pesar de su importancia, presenta una serie de limitantes tecnológicas y socioeconómicas; su producción se desarrolla principalmente en la región Andina, en laderas de las cordilleras, entre 700 y $2000 \mathrm{msnm}$, donde las condiciones fisiográficas son muy variables y predominan áreas de pendientes que varían entre el $10 \%$ y $40 \%$, lo que expone el suelo a un alto riesgo de degradación (Volverás y Amézquita, 2009). La problemática genera baja productividad tanto agrícola como de proceso y deficiencia en la calidad del producto, en Nariño, afecta a cerca del $50 \%$ de las unidades productivas (Mafla, 1982; Rodríguez, 2000), de tal manera que mientras algunas zonas productoras del país obtuvieron hasta $12 \mathrm{t} \mathrm{ha}^{-1}$ de panela o (ANC), Nariño solo alcanzó 6 t ha ${ }^{-1}$ (Rangel et al., 2006), lo que sugiere que existe una posibilidad de mejorar la productividad del sistema a través del ajuste de algunas prácticas de manejo del cultivo. Parte de problemática está relacionada con la práctica de fertilización y los sistemas de siembra que tienen diversas condiciones, lo cual ha generado un sinnúmero de recomendaciones que en muchos casos afectan el entorno ambiental, la rentabilidad del cultivo y la calidad del producto (Buenaventura y Rangel, 1976; Manrique et al., 2000; Requier-Desjardins y Rodríguez, 2004; DANE, 2017).

La nutrición mineral y la aplicación de fertilizantes en caña de azúcar para panela o ANC, es un aspecto técnico que no se ha abordado con la debida importancia en el país y la mayoría de los trabajos reportan sobre la producción de azúcar (Martínez, 2013). En la general, la mayor parte de los productores fertiliza, sin embargo, sus dosis varían inclusive de un lote a otro, también dependen del costo relativo de los fertilizantes y el precio de comercialización del azúcar no centrifugado (Mendez, 1981; Rangel et al., 2006). Para diferentes zonas paneleras, la práctica de fertilización va desde no utilizar ningún tipo de abono hasta aplicar entre 50 y $500 \mathrm{~kg} \mathrm{ha}^{-1}$ de algún material compostado, y por último, usar entre 250 y $500 \mathrm{~kg} \mathrm{ha}^{-1}$ de fertilizante mineral N-P-K, hasta la mezcla de las dos fuentes, sin considerar varios aspectos ecofisiológicos determinantes en el proceso de nutrición vegetal para obtener máximos rendimientos, calidad y mantener la productividad del suelo (Marschner, 1995; Loneragan, 1997; Palled, 2011; Bonilla, 2008).

En otros países productores, los trabajos sobre fertilización y uso de abono orgánico para la producción de panela o ANC y azúcar, reportan múltiples resultados. A nivel internacional en la región de Pakistán, Khan et al. (2005) al evaluar diferentes niveles de N-P-K, encontraron que el mayor rendimiento de caña y azúcar, se obtuvo con 200, 120 y $150 \mathrm{~kg} \mathrm{ha}^{-1}$ de N, $\mathrm{P}_{2} \mathrm{O}_{5}$ y K $\mathrm{K}_{2}$. En un trabajo sobre micronutrientes en el estado de Sao Paulo (Brasil), se observó que el rendimiento de la caña de azúcar aumentó significativamente, con zinc ( $\mathrm{Zn}$ ) aumentaron 18 t ha-1 de caña, con molibdeno (Mo) y manganeso (Mn) llegaron a $12 \mathrm{t} \mathrm{ha}^{-1}$ de caña, con cobre (Cu) alcanzaron $10 \mathrm{t} \mathrm{ha}^{-1} \mathrm{de}$ caña, con boro (B) $9 \mathrm{t} \mathrm{ha}^{-1}$ de caña y con todos los micronutrientes $9 \mathrm{t} \mathrm{ha}^{-1}$ de caña, finalmente tuvieron este orden de magnitud, $\mathrm{Zn}>\mathrm{Mo}=\mathrm{Mn}>\mathrm{Cu}>\mathrm{B}=$ completo (Mellis et al., 2016). En la India, para una producción de $100 \mathrm{t} \mathrm{ha}^{-1} \mathrm{la}$ planta extrajo de macronutrientes 140,34 y $332 \mathrm{~kg} \mathrm{ha}^{-1} \mathrm{de} \mathrm{N}-\mathrm{P}-\mathrm{K}$, y en orden decreciente los nutrientes con mayor demanda por la caña de azúcar fueron $\mathrm{K}>\mathrm{N}>\mathrm{Ca}>\mathrm{Mg}>\mathrm{P}$ (Dotaniya et al., 2016).

En Colombia en el departamento de Antioquia, se encontró que los mayores rendimientos de caña se dieron con dosis que variaron entre 50 y $75 \mathrm{~kg} \mathrm{ha}^{-1}$ de $\mathrm{N}, 75$ a $150 \mathrm{~kg} \mathrm{ha}^{-1} \mathrm{de}_{2} \mathrm{O}_{5}$ y 75 a $225 \mathrm{~kg} \mathrm{ha}^{-1}$ de $\mathrm{K}_{2} \mathrm{O}$ (Muñoz, 1978). Por otro lado, se reportó que $50 \mathrm{t}$ de caña de azúcar para panela o ANC extrajeron $34 \mathrm{~kg} \mathrm{~N}, 23 \mathrm{~kg} \mathrm{P}_{2} \mathrm{O}_{5}$ y $68 \mathrm{~kg}$ de $\mathrm{K}_{2} \mathrm{O}$, con aplicaciones al momento de la siembra y entre 30 - 40 días después de cada corte (Osorio, 2007); en la meseta de Popayán, de igual manera se evidenciaron que los mejores resultados en rendimiento de caña y panela o ANC se obtuvieron con 100, 90 y $30 \mathrm{~kg} \mathrm{ha}^{-1}$ de N, $\mathrm{P}_{2} \mathrm{O}_{5}$ y $\mathrm{K}_{2} \mathrm{O}$, respectivamente (Torres et al., 1998); mientras que en la zona de la Hoya del Rio Suárez (HRS), Manrique et al. (2000) reportaron que en caña monocultivo los mejores rendimientos se obtuvieron con niveles de 100, 200 y $100 \mathrm{~kg} \mathrm{ha}^{-1}$ de N, $\mathrm{P}_{2} \mathrm{O}_{5}$ y $\mathrm{K}_{2} \mathrm{O}$, respectivamente.

En la mayoría de los países productores de panela o ANC, también se utilizan abonos orgánicos en la práctica de fertilización (Shivanaikar, 2012). En Bangalore (India), Palled (2011) encontró mejor comportamiento agronómico y rendimiento cuando usó $50 \%$ de N químico y $50 \%$ de N proveniente de residuos de la caña; en Colombia se estimó que cerca del $24 \%$ de los productores de caña de azúcar para panela o ANC utilizan algún tipo 
de abono orgánico como gallinaza, ceniza de la hornilla o compost en diferentes dosificaciones, práctica derivada por la variación constante de los precios de la panela o ANC y de los fertilizantes de síntesis química.

Los agricultores sostienen que el abono orgánico es importante porque incrementa el rendimiento, la calidad de la panela o ANC y sustituye fertilizantes minerales para disminuir costos de producción (Rangel et al., 2006). Es importante conocer los efectos de los insumos utilizados en la producción de caña de azúcar para panela o ANC, ya que proporcionar una cantidad elevada de nutrientes puede causar disminución en productividad o reducción en un nivel específico de nutrientes en la planta (Borges et al., 2015), De acuerdo con Latief et al. (2010), la aplicación de fertilizantes orgánicos ha tenido favorable comportamiento comparado con el uso de fertilizantes químicos, sin embargo, es fundamental el uso equilibrado de insumos orgánicos, inorgánicos y biofertilizantes para mantener la armonía del ambiente físico, químico y biológico del suelo (Gopalasundaram et al., 2012).

En algunas regiones de Colombia, se encontró un efecto en el rendimiento de caña con dosis de $3 \mathrm{t}_{\text {ha }}{ }^{-1}$ de abono orgánico complementado con N, P y K (Manrique et al., 2000). Al evaluar diferentes dosis de compost en combinación con fertilizantes minerales, se reportó que $5 \mathrm{t} \mathrm{ha}^{-1}$ de compost mejoraron la productividad del cultivo de caña (Muñoz, 2012). Por su parte Maldonado et al. (2006), mencionaron que con $5 \mathrm{t} \mathrm{ha}^{-1} \mathrm{de}$ compost elaborado en la finca se alcanzaron mejoras en el rendimiento de caña de azúcar en pequeñas fincas. La aplicación de $200 \mathrm{t}$ $\mathrm{ha}^{-1}$ de cachaza compostada o $10 \mathrm{t} \mathrm{ha}^{-1}$ de cenichaza (ceniza + cachaza), lograron grandes mejoras en rendimiento de caña y azúcar e incrementos en contenidos de nutrientes del suelo como P (Quintero, 1995), sin embargo, al comparar tratamientos de fertilización mineral y abono orgánico, se encontró que aplicando solo abono orgánico se disminuyó drásticamente el desarrollo de la planta de caña (García et al., 2010).

El agricultor o productor de panela o ANC, realiza prácticas para mejorar el rendimiento de la caña, una de ellas y de gran importancia es la nutrición adecuada, ya que no solo mejora el rendimiento si no que mejora la calidad del producto. Aplicaciones deficientes o no balanceadas de nutrientes pueden reducir hasta $52 \%$ del rendimiento de caña por hectárea y el rendimiento de sacarosa (Quintero, 1995). La sola aplicación de N, P y $\mathrm{K}$, además de no suministrar todos los nutrientes necesarios para un óptimo rendimiento, genera deficiencias de nutrientes que constantemente el cultivo extrae y no se reemplazan como B, Cu, Fe, $\mathrm{Mn}$ y $\mathrm{Zn}$. Bajo esquemas de producción intensivos, es posible conseguir buenos rendimientos en caña y panela o ANC a partir de fertilizantes minerales, con un adecuado uso de abonos orgánicos.

El sistema de siembra presenta un papel importante sobre la productividad en el cultivo de caña de azúcar para panela o ANC, el mateado o espaciado (SE) se utiliza en las zonas donde se realiza el corte de caña por entresaque o desguíe. El sistema de siembra consiste en sembrar la caña, preferiblemente cogollo, en cajuelas elaboradas a una distancia aproximada de 50 a $80 \mathrm{~cm}$ entre sí, la cantidad de cogollos que se ubican en cada orificio varía entre dos a cuatro, sin conservar un surco como tal. La caña se ubica con una leve inclinación en el orificio, dejando la zona apical visible. Este sistema requiere de gran cantidad de semilla, aproximadamente entre 12 y $14 \mathrm{t} \mathrm{ha}^{-1}$ por hectárea (Manrique et al., 2010). En el sistema de siembra denominado estaca continúa (SEC), que comprende surcos con una distancia entre 1,20 m a 1,30 m entre sí, se distribuye la semilla en trozos largos en el fondo del surco, posteriormente se corta con machete o herramienta de corte, en trozos de tres a cuatro yemas. De acuerdo con la disponibilidad de semilla y calidad de esta, se puede cruzar la parte basal con la parte apical (punta con pata), de lo contrario, simplemente se riega sin traslape (García et al., 2007).

En algunas zonas productoras de Colombia se ha encontrado que el sistema espaciado puede reducir hasta en $15 \%$ la producción de caña, cuando se presenta déficit hídrico y desigualdad en el crecimiento de los tallos y mayor riesgo de volcamiento en comparación con el sistema estaca continúa, sin embargo, dependiendo de las condiciones de suelo, topografía y material vegetal disponible, se puede encontrar ventajas de un sistema respecto al otro (Manrique, 1992; Manrique et al., 2000). En sistemas de siembra estaca continúa y espaciado se reportó que el primero presentó los mejores resultados en producción de caña, con distancias de 1,40 a 1,50 m entre surcos, mientras que en el espaciado con distancias entre 1 y $1,30 \mathrm{~m}$ entre surcos y de 25 a $50 \mathrm{~cm}$ entre plantas, con uno y dos esquejes por sitio, obtuvo rendimientos cercanos al sistema de siembra estaca continúa (Estupiñán et al., 2000). 
El objetivo del presente trabajo fue evaluar el uso de fertilizante orgánico y mineral sobre el rendimiento de la producción de caña de azúcar para panela o azúcar no centrifugada (ANC) en su primer ciclo de producción.

\section{Materiales y métodos}

\section{Localización}

La investigación se desarrolló entre los meses de noviembre de 2008 y el mes de Marzo de 2010, en la vereda Villa Inés, corregimiento Olaya Herrera del municipio de Consacá ( $01^{\circ} 15^{\prime}$ 68,8” N; $77^{\circ}$ 29’37,1” W), Región Andina, Macizo del Galeras, departamento de Nariño (Colombia). En esta zona predomina el clima medio húmedo, temperatura media anual de $17{ }^{\circ} \mathrm{C}, 1700 \mathrm{~mm}$ de precipitación media anual, $86 \%$ de humedad relativa, $1650 \mathrm{msnm}$, pendiente media de $18 \%$, suelo clasificado como Humic Eutrudepts, litología y sedimentos de rocas volcánicas piroclásticas consolidadas (IGAC, 2004) y condiciones de fertilidad química media-baja (Cuadro 1).

Cuadro 1. Descripción de las condiciones químicas del suelo, vereda Villa Inés, municipio Consacá. Nariño, Colombia. 2008-2010. Table 1. Description of the chemical conditions of the soil, rural area of Villa Ines, Consacá municipality. Nariño, Colombia. 20082010 .

\begin{tabular}{|c|c|c|c|c|c|c|c|c|c|c|c|c|c|c|}
\hline $\mathbf{P}$ & $\mathbf{S}$ & $\mathrm{Fe}$ & $\mathrm{Cu}$ & Mn & $\mathbf{Z n}$ & B & \multirow{2}{*}{$\mathbf{p H}$} & MO & $\mathbf{A l}+\mathbf{H}$ & Al & $\mathbf{C a}$ & Mg & $\mathbf{K}$ & $\mathbf{N a}$ \\
\hline \multicolumn{7}{|c|}{$\mathrm{mg} \mathrm{kg}^{-1}$} & & $\%$ & \multicolumn{6}{|c|}{ Cmol (+) kg-1 } \\
\hline 4 & 8,5 & 403 & 4,8 & 43,7 & 2,1 & 0,11 & 5,7 & 2,9 & 0,19 & 0 & 6,86 & 2,21 & 0,81 & 0,11 \\
\hline
\end{tabular}

* Laboratorios de la Corporación Colombiana de Investigación Agropecuaria (AGROSAVIA) / Laboratories of the Corporación Colombiana de Investigación Agropecuaria (AGROSAVIA).

\section{Materiales evaluados}

Se utilizó el genotipo de caña de azúcar Canal Point (CP 782018), conocido comúnmente en la región como palmireña (Figura 1), es una variedad de porte alto de buen macollamiento. Posee tallos color verdoso con cerosina, presenta deshoje natural parcial, tallos con entrenudos de diámetro y longitud homogénea e intermedia, muestra abundante follaje sin pubescencia y pocas lalas (Aguiar, 2001).

\section{Diseño experimental}

Se estableció un experimento con un diseño de bloques completamente al azar (DBCA) con nueve tratamientos y tres repeticiones; los tratamientos consideraron sistema de siembra y niveles de fertilización N-P-K, ajustados según análisis de suelo. Cada unidad experimental (UE) estuvo constituida por seis surcos de seis metros de largo establecidos a una distancia de $1,3 \mathrm{~m}$ para un área de $39 \mathrm{~m}^{2}$. Tratamientos: T1: estaca continúa a $1,30 \mathrm{~m}$ entre surcos con fertilización del agricultor; T2: estaca continúa a 1,30 m entre surcos con abono orgánico según análisis de suelos; T3: estaca continúa a 1,30 m entre surcos con fertilización química según análisis de suelos; T4: estaca continúa a 1,30 m entre surcos con mezcla de fertilización química y abono orgánico según análisis de suelos; T5: sistema espaciado según análisis de suelo; T7: sistema espaciado con mezcla de fertilización química y abono orgánico según análisis de suelos; T8: sistema espaciado con fertilización del agricultor; T9: sistema estaca continúa y espaciado sin fertilización como testigo. 


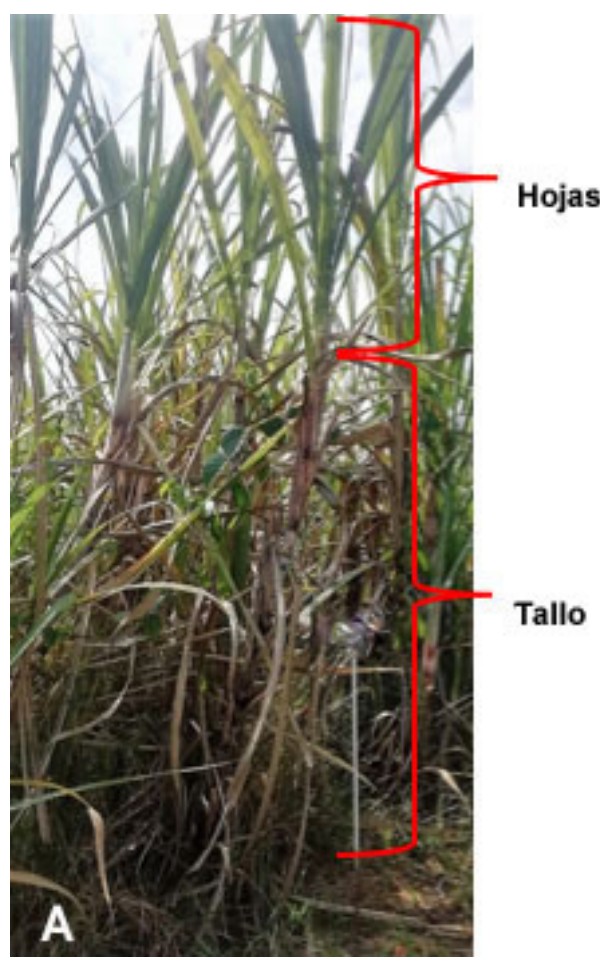

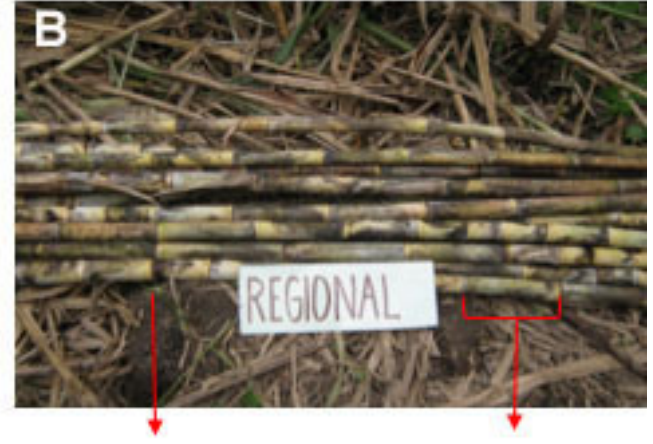

Nudo

\section{Entrenudo}

Figura 1. Variedad de caña de azúcar para panela Canal Point (CP 782018). A. Partes aéreas (tallo y hojas). B. Partes del tallo en caña de azúcar (entrenudo y nudo). Nariño, Colombia. 2008-2009.

Figure 1. Variety of sugarcane for Canal Point panela (CP 782018). A. Aerial parts (stem and leaves. B. Parts of the stem in sugarcane (internode and knot). Nariño, Colombia. 2008-2009.

\section{Manejo agronómico}

Las fuentes y nutrientes por tratamiento, según análisis de suelo (Cuadro 2), fueron las siguientes: para los tratamientos fertilización química del agricultor se utilizaron $550 \mathrm{~kg} \mathrm{ha}^{-1}$ de un fertilizante compuesto con la fórmula (13-26-6), que es la fuente y cantidad que usualmente utilizan los agricultores de la zona; para los

Cuadro 2. Cantidad de nutrientes nitrógeno $(\mathrm{N})$, fósforo $\left(\mathrm{P}_{2} \mathrm{O}_{5}\right)$ y Potasio $\left(\mathrm{K}_{2} \mathrm{O}\right)$, para los tratamientos de fertilización en caña de azúcar (Saccharum officinarum L.), definidos según el análisis de suelo. Nariño, Colombia. 2008-2010.

Table 2. Amount of nutrients nitrogen $(\mathrm{N})$, phosphorus $\left(\mathrm{P}_{2} \mathrm{O}_{5}\right)$, and potassium $\left(\mathrm{K}^{2} \mathrm{O}\right)$ for fertilization treatments in sugarcane (Saccharum officinarum L.), defined according to the soil analysis. Nariño, Colombia. 2008-2010.

\begin{tabular}{lccc}
\hline Descripción & $\mathbf{N}$ & $\mathbf{P}_{\mathbf{2}} \mathbf{O}_{\mathbf{5}}$ & $\mathbf{K}_{\mathbf{2}} \mathbf{O}$ \\
\cline { 2 - 4 } & & $\mathbf{~ k g ~ h a}^{\mathbf{1}}$ & \\
\hline Abono orgánico & 27 & 45 & 18,8 \\
Fertilización química del agricultor & 71,5 & 143 & 33 \\
Mezcla de abono orgánico y fertilizante químico & 96 & 57 & 39,4 \\
Fertilización química según análisis de suelo & 165 & 69 & 60 \\
\hline
\end{tabular}


tratamientos fertilización química según análisis de suelo se utilizó urea (46-0-0), fosfato diamónico (18-46-0) y cloruro de potasio (0-0-60); en los tratamientos con abono orgánico se utilizaron $1500 \mathrm{~kg} \mathrm{ha}^{-1}$ de un fertilizante orgánico con contenido de nitrógeno $(1,8 \%)$, fósforo $\left(\mathrm{P}_{2} \mathrm{O}_{5}\right)(3,0 \%)$, potasio $\left(\mathrm{K}_{2} \mathrm{O}\right)(1,25 \%)$, carbono orgánico $(18,1 \%)$, cenizas $(41,1 \%)$ y relación C/N (10 \%) (Cuadro 3). Para los tratamientos de mezcla fertilización química y abono orgánico las dosis correspondieron al $50 \%$ de las cantidades de los tratamientos individuales, teniendo en cuenta que cuando el productor utiliza abono orgánico disminuye la cantidad de fertilizante químico.

Cuadro 3. Contenido químico del abono orgánico, empleado como parte del tratamiento de fertilización en caña de azúcar (Saccharum officinarum L.), a nivel experimental. Nariño, Colombia. 2008-2010.

Table 3. Chemical content of organic fertilizer, used as a part of the fertilization treatment in sugar cane (Saccharum officinsrum L.), at an experimental level. Nariño, Colombia. 2008-2010.

\begin{tabular}{cc}
\hline Componente & Cantidad (\%) \\
\hline Nitrógeno total $(\%)$ & 1,8 \\
Fosforo $\left(\mathrm{P}_{2} \mathrm{O}_{5}\right)$ & 3,0 \\
Potasio $\left(\mathrm{K}_{2} \mathrm{O}\right)$ & 1,25 \\
Carbono orgánico & 18,1 \\
Cenizas & 41,1 \\
Relación C/N & 10 \\
\hline
\end{tabular}

\section{Variables evaluadas}

Una vez alcanzado el índice de madurez (IDM), que es la relación entre los sólidos solubles totales (SST) de la parte apical (SST apical) y basal (SST basal) del tallo, es decir, la división entre SST apical y SST basal, se consideró que la caña se encontraba adecuada para cosechar, cuando presentó un resultado entre 0,91 a 1,00, siendo importante para la programación de la molienda experimental (Larrahondo y Villegas, 1995).

\section{Rendimiento de caña}

Al determinar que la caña se encuentra adecuada para cosechar, se hizo la evaluación en dos metros lineales en tres puntos de unidad de muestreo, contando el número de tallos presentes y luego fueron cosechados para determinar la masa fresca. Luego se determinó la variable en toneladas por hectárea (TCH), de acuerdo con la siguiente ecuación (1):

$\mathrm{TCH}=\left(\frac{\text { Masa fresca de los tallos por } 2 \mathrm{~m} \text { lineales } \mathrm{x} 7692,3^{1}}{2}\right) / 1000000$

${ }^{1}$ metros lineales que tiene una hectárea de terreno con la distancia de siembra de 1,3 m entre surcos.

\section{Rendimiento de panela o (ANC)}

Para la determinación de esta variable se efectuó la molienda experimental, la cual consistió en la transformación de caña a panela o ANC, con el procedimiento descrito por Guaman et al. (2009), en el cual una vez 
que las unidades experimentales en conjunto alcanzaron el IDM (valor comprendido entre 0,91 y 1,00), se realizó la cosecha, y se colectó aproximadamente $15 \mathrm{~kg}$ de caña por cada UE, debidamente identificadas; seguidamente se transportó de campo al trapiche para continuar el proceso poscosecha. Previo al proceso de extracción del jugo se realizó el pesaje de la caña colectada en campo de manera individual para determinar el valor exacto de la caña por cada UE, posteriormente se extrajo el jugo de la caña a través del molino (R14 El Panelero de 2,0 t caña ha ${ }^{-1}$ ), de la totalidad obtenida se extrajeron $4 \mathrm{~kg}$ de jugo, enseguida se pasaron a recipientes metálicos debidamente tarados, e inició el calentamiento del jugo (que comprende desde jugo crudo hasta la obtención de miel) y los siguientes procesos para transformación (Figura 2).

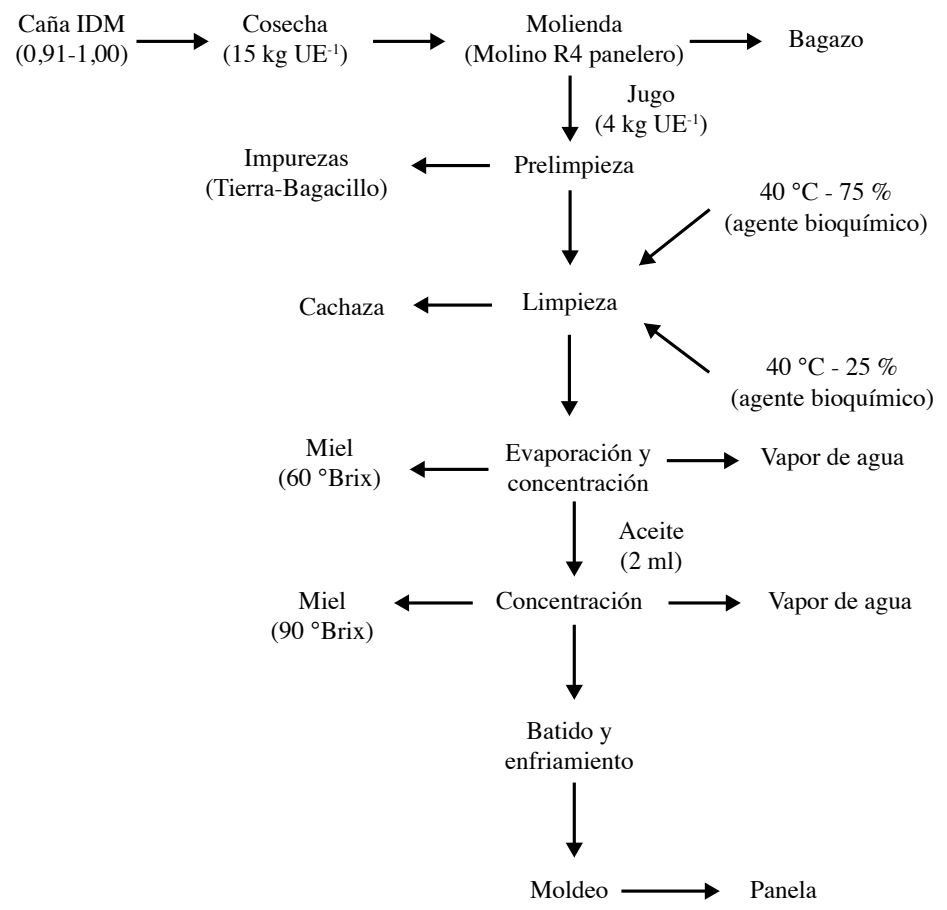

Figura 2. Diagrama del proceso de elaboración de panela o azúcar no centrifugado (ANC). Nariño, Colombia. 2008-2010.

Figure 2. Diagram of the process of making non-centrifuged sugar (NCS). Nariño, Colombia. 2008-2010.

1. Prelimpieza, consiste en extraer por medios físicos algunas partículas presentes como tierra (decantación) y bagacillo (flotación) / Pre-cleaning, consists of extracting by physical means some particles present such as soild (settiling) and bagasse (flotation).

2. Limpieza, por medio de calor y agentes bioquímicos (guácimo, cadillo o balso), que floculan y aglutinan, se obtuvo el subproducto denominado cachaza / Cleaning, by means of heat and biochemical agents (guacimo, cadillo or balso) which flocculate and agglutinate the by-product called cachaça was obtained.

3. Evaporación y concentración, consiste en eliminar el alto contenido de agua del jugo mediante calor, de lo cual resulta la miel, con un valor aproximado de $60^{\circ}$ Brix / Evaporation and concentration, consists of eliminating the high water content of the juice by means of heat, resulting in honey, with and approximate value of $60^{\circ}$ Brix.

4. Concentración de miel, se continúa calentando la miel extrayendo más agua, a un punto donde los SST obtienen un valor cercano a los $90^{\circ} \mathrm{Brix}$, a fin de dar punto a la miel / Honey concentration, the honey continues to be heated extracting more water, to a point where the TSS obstain a value close to $90^{\circ}$ Brix, in order to give the honey a point.

5. Batido, la miel se pasa a una bandeja especial donde se realiza el batido con ayuda de palas a fin de enfriarla / Smoothie, the honey is transferred to a special tray where the smoothie is made with the help of shovels in order to cool it down.

6. Moldeo, se vierte la miel después del proceso anterior en moldes de madera, con capacidad de 1 lb para así obtener la panela o ANC / Molding, the honey is poured after the previous process in wooden molds, with a capacity of 1lb to obtain the panela or NCS. 
La caña colectada en campo, el jugo obtenido por la caña y la miel en proceso de enfriamiento (panela o ANC), se pesaron y anotaron de manera individual, así por cada UE, en un formato construido para tal fin; consecutivamente se hizo el procesamiento de datos para determinar la caña requerida para la obtención de los $4 \mathrm{~kg}$ de jugo que fueron procesados (ecuación 2), una vez determinado este valor se procedió a calcular el porcentaje de conversión de caña a panela o ANC (ecuación 3) y luego las toneladas de panela por hectárea (TPH) (ecuación 4).

$$
\begin{aligned}
& \text { Caña requerida }\left(\mathrm{kg} 4 \mathrm{~kg} \text { de jugo }{ }^{-1}\right)=\left(\frac{4 \mathrm{~kg} \text { de jugo x Peso de la caña por UE }(\mathrm{kg})}{\text { Jugo obtenido por UE }(\mathrm{kg})}\right) \\
& \text { Conversión de caña a panela }(\%)=\left(\frac{\text { Panela obtenida por UE }(\mathrm{kg})}{\text { Caña requerida }\left(\mathrm{kg} 4 \mathrm{~kg} \mathrm{de} \mathrm{jug0^{-1 } )}\right.}\right) \times 100 \\
& \mathrm{TPH}=\left(\frac{\text { Toneladas de caña por hectárea }(\mathrm{TCH}) \times \text { Conversión de caña a panela }(\%)}{100}\right)
\end{aligned}
$$

\section{Análisis fisicoquímicos en laboratorio}

\section{Azúcares reductores (\%)}

Para la determinación de los azúcares reductores se ajustó el método volumétrico de Lane y Eynon (1923) de la siguiente manera: se pesaron $10 \mathrm{~g}$ de panela o ANC previamente rallada y se agregó $50 \mathrm{ml}$ de agua hasta que la mezcla estuviese totalmente diluida. Luego, esta dilución se llevó a un balón aforado de $100 \mathrm{ml}$ y se completó el volumen con agua destilada para obtener una dilución 1:10 p/v. Posteriormente, se transfirió esta solución de panela o ANC a una bureta de $25 \mathrm{ml}$ para efectuar la titulación, seguidamente en un erlenmeyer se añadió en orden, $5 \mathrm{ml}$ de la solución Fehling B, $5 \mathrm{ml}$ de la solución Fehling A, tres gotas de aceite mineral y desde la bureta se agregaron de 2 a $3 \mathrm{ml}$ de la solución de panela o ANC. Luego se llevó esta mezcla a ebullición y se contabilizaron exactamente 2 min, tiempo preciso para agregar dos gotas del indicador azul de metileno y continuar la titulación dejando caer gota a gota a la muestra hasta que el color azul haya desaparecido completamente. Con el volumen gastado se realizaron los cálculos para expresar el resultado como \% de azúcares reductores (ecuación 5).

$$
\text { Azúcares reductores }(\%)=\left(\frac{\text { Factor Fehling }}{\text { Volumen gastado (ml) }}\right) \times \text { Factor de dilución (FD) }
$$

\section{Fosfatos en panela o azúcar no centrifugada $(A N C)(\mathrm{ppm})$}

La medición de los fosfatos en panela o ANC se obtuvo a través del método colorimétrico 4 500-P E (Eaton et al., 2005). Para el análisis se pesaron $5 \mathrm{~g}$ de panela o ANC y se llevaron a un volumen con agua destilada de $100 \mathrm{ml}$ (dilución 1:20 p/v), a continuación se prepararon dos soluciones, la solución A, compuesta por molibdato de amonio, tartrato de antimonio y potasio, y ácido sulfúrico, y B que era una solución de ácido ascórbico; con ambas se preparó la solución de trabajo, con $45 \mathrm{ml}$ de la solución A y $800 \mathrm{ml}$ de agua destilada, se mezcló hasta homogenizar y se agregó $18 \mathrm{ml}$ de la solución B, aforando hasta 1 litro con agua destilada; luego de la dilución de la muestra, se tomó $1 \mathrm{ml}$ y se agregaron $9 \mathrm{ml}$ de la solución de trabajo (solución A + solución B), se dejó en reposo por 15 min para que desarrollara el color y se realizó la lectura en el espectrofotómetro a $670 \mathrm{~nm}$, se empleó como 
referencia agua destilada; previamente se debió montar la curva patrón con mínimo cinco puntos de concentración, utilizando dihidrógeno fosfato de potasio $\left(\mathrm{KH}_{2} \mathrm{PO}_{4}\right)$; para el cálculo se utilizó la ecuación 6 y los resultados se reportaron en partes por millón (ppm) de $\mathrm{P}_{2} \mathrm{O}_{5}$,

$$
\text { Fosfatos }\left(\mathrm{ppm}_{2} \mathrm{O}_{5}\right)=\text { Factor de dilución }(\mathrm{FD}) \times \text { Lectura en espectrofotométro }
$$

Los datos obtenidos de cada variable fueron sometidos a los supuestos de normalidad y de homogeneidad; luego se hizo el análisis de varianza (anova), mediante el programa SAS 9.4; se utilizaron pruebas de comparación múltiple Tukey, para determinar el efecto de los tratamientos sobre las variables evaluadas, con un nivel de confiabilidad del $95 \%$.

\section{Resultados}

\section{Rendimiento de caña de azúcar para panela o azúcar no centrifugada (ANC)}

El uso de abono orgánico y la fertilización mineral tuvo un efecto significativo sobre el rendimiento de caña ( $\mathrm{p}<0,05)$, los tratamientos (T3) estaca continua con fertilización química con 168,20 TCH, (T1) estaca continua con fertilización del agricultor con 167,07 TCH y (T5) espaciado con abono orgánico con 167 TCH presentaron el mayor rendimiento de caña con respecto a los demás tratamientos, y fueron significativamente diferentes con respecto al tratamiento (T9) estaca continua y espaciado sin fertilización con 127,47. Los tratamientos de menor rendimiento fueron los que correspondieron (T8) al sistema de siembra espaciado con fertilización del agricultor con 134,1 TCH y el testigo (T9) con 127,47 TCH, en tanto a los tratamientos que combinaron el sistema de siembra espaciado y los sistemas de fertilización, los mayores rendimientos de caña se obtuvieron con abono orgánico (T5) y fertilización química (T6) con 167 y 156 TCH, respectivamente (Cuadro 4).

Cuadro 4. Diferencias entre tratamientos de fertilización orgánica y química, para el rendimiento de caña de azúcar (Saccharum officinarum L.) (t ha ${ }^{-1}$ ), en el municipio de Consacá. Nariño, Colombia. 2008-2010.

Table 4. Differences between organic and chemical fertilization treatments for the cane (Saccharum officinarum $\mathrm{L}$.) yield ( $\mathrm{t}$ ha-1), in the municipality of Consacá. Nariño, Colombia. 2008-2010.

\begin{tabular}{lc}
\hline Tratamientos & Caña $\left(\mathbf{t ~ h a}^{-1}\right)^{*}$ \\
\hline T1: estaca continúa con fertilización del agricultor & $167,07 \mathrm{a}$ \\
T2: estaca continúa con abono orgánico & $148,20 \mathrm{bc}$ \\
T3: estaca continúa con fertilización química & $168,20 \mathrm{a}$ \\
T4: estaca continúa mezcla fertilización química/abono orgánico & $144,50 \mathrm{c}$ \\
T5: espaciado con abono orgánico & $167,00 \mathrm{a}$ \\
T6: espaciado con fertilización química & $156,30 \mathrm{~b}$ \\
T7: espaciado mezcla fertilización química/abono orgánico & $144,33 \mathrm{c}$ \\
T8: espaciado con fertilización del agricultor & $134,10 \mathrm{~d}$ \\
T9: estaca continúa y espaciado sin fertilización & $127,47 \mathrm{de}$ \\
\hline
\end{tabular}

*Promedios con la misma letra no son diferentes estadísticamente $(\mathrm{p}<0,05) / *$ Averages with the same letter are not statistically different $(\mathrm{p}<0.05)$. 


\section{Rendimiento de panela o azúcar no centrifugada (ANC)}

Los rendimientos en panela o (ANC), medidos como la capacidad de conversión de caña de azúcar a panela o ANC y las toneladas de panela o ANC producidas, tuvieron diferencias estadísticas $(\mathrm{p}<0,05)$. Para conversión a panela o ANC el mejor resultado fue $12,1 \%$ en el tratamiento sistema de siembra espaciado con fertilización química (T6) y difirió de los demás tratamientos a excepción del tratamiento sistema de siembra espaciado con fertilización del agricultor (T8) que tuvo 11,73\%; el menor valor de conversión lo presentó el tratamiento sistema de siembra estaca continúa con fertilización del agricultor (T1) con 9,49\%; por otra parte, la interpretación de estos resultados se explica de la siguiente manera, por cada $100 \mathrm{TCH}$ se obtuvieron 12,1 TPH.

En relación con las toneladas de panela o ANC por hectárea (TPH), los mayores valores se obtuvieron en los tratamientos con fertilización química, tanto en sistema de siembra espaciado (T6) (18,78 TPH) como en el sistema de siembra estaca continúa (17,64 TPH), los cuales solo tuvieron diferencias estadísticas con el tratamiento testigo, sistema de siembra estaca continúa y espaciado sin fertilización (T9) que obtuvo 13,06 TPH, el menor valor de producción (Cuadro 5).

Cuadro 5. Diferencias entre tratamientos de fertilización orgánica y química, para conversión a panela o azúcar no centrifugado (\%) y rendimiento de caña de azúcar (Saccharum officinarum L.) ( $\mathrm{t}$ ha ${ }^{-1}$ ) en el municipio de Consacá. Nariño, Colombia. 2008-2010.

Table 5. Differences between organic and chemical fertilization treatments, for conversion to panela or non-centrifugal sugar (\%) and sugarcane yield (Saccharum officinarum L.) ( $\left.\mathrm{t} \mathrm{ha}^{-1}\right)$ in the municipality of Consacá. Nariño, Colombia. 2008-2010.

\begin{tabular}{lcc}
\hline Tratamientos & Conversión $(\%)^{*}$ & Panela $\left(\mathbf{t}\right.$ ha-1)* $^{*}$ \\
\hline T1: estaca continúa con fertilización del agricultor & $9,49 \mathrm{e}$ & $16,16 \mathrm{ab}$ \\
T2: estaca continúa con abono orgánico & $10,24 \mathrm{~d}$ & $15,19 \mathrm{ab}$ \\
T3: estaca continúa con fertilización química & $10,49 \mathrm{~cd}$ & $17,64 \mathrm{a}$ \\
T4: estaca continúa mezcla fertilización química/abono orgánico & $11,38 \mathrm{~b}$ & $16,44 \mathrm{ab}$ \\
T5: espaciado con abono orgánico & $10,28 \mathrm{~d}$ & $17,17 \mathrm{ab}$ \\
T6: espaciado con fertilización química & $12,10 \mathrm{a}$ & $18,78 \mathrm{a}$ \\
T7: espaciado mezcla fertilización química/abono orgánico & $11,23 \mathrm{bc}$ & $16,21 \mathrm{ab}$ \\
T8: espaciado con fertilización del agricultor & $11,73 \mathrm{ab}$ & $15,73 \mathrm{ab}$ \\
T9: estaca continúa y espaciado sin fertilización & $10,25 \mathrm{~d}$ & $13,06 \mathrm{~b}$ \\
\hline
\end{tabular}

*Promedios con la misma letra no son diferentes estadísticamente $(\mathrm{p}<0,05) / *$ Averages with the same letter are not statistically different $(\mathrm{p}<0.05)$.

\section{Análisis fisicoquímicos en laboratorio}

En la determinación de azúcares reductores (\%), el tratamiento sistema de siembra espaciado con fertilización química del agricultor (T8) obtuvo 13,2\%, fue el mayor valor y presentó diferencias estadísticas altamente significativas $(\mathrm{p}<0,01)$ con los tratamientos restantes, a excepción de los tratamientos sistema de siembra espaciado con fertilización del agricultor (T6) que presentó $12,1 \%$ y el tratamiento testigo (T9) con $12 \%$. Los menores valores los presentaron el sistema de siembra estaca continúa con mezcla de fertilización química/abono orgánico (T4) que tuvo 8,5 \%, sistema de siembra estaca continúa con fertilización del agricultor (T1) con 9,5 \% y fertilización química (T3) con 9,9\% (Cuadro 6). 
Para los fosfatos (ppm) en panela o ANC, el mayor contenido de fósforo se obtuvo en el testigo (T9) con 753 ppm, seguido por estaca continúa con abono orgánico (T2) que presento $706 \mathrm{ppm}$, los cuales tuvieron diferencias altamente significativas $(\mathrm{p}<0,01)$ con los demás tratamientos. Los menores contenidos se presentaron en estaca continúa con fertilización química del agricultor (T1) con 514 ppm y con fertilización química (T3) que tuvo 543 ppm; el fósforo en los demás tratamientos fue similar y estuvo entre 561 y 595 ppm (Cuadro 6).

Cuadro 6. Diferencias entre tratamientos de fertilización orgánica y química para los azúcares reductores (\%) y fosfatos en panela o (ANC) de caña de azúcar (Saccharum officinarum L.), del municipio de Consacá. Nariño, Colombia. 2008-2010.

Table 6. Differences between organic and chemical fertilization treatments for reducing sugars (\%) and phosphates in panela or noncentrifugal sugar cane (Saccharum officinarum L.) of the municipality of Consacá. Nariño, Colombia. 2008-2010.

\begin{tabular}{lcc}
\hline Tratamientos** & $\begin{array}{c}\text { Azúcares reductores en } \\
\text { panela }(\%) *\end{array}$ & Fosfatos en panela (ppm)* \\
\hline T1: estaca continúa con fertilización del agricultor & $9,50 \mathrm{de}$ & $514 \mathrm{~d}$ \\
T2: estaca continúa con abono orgánico & $11,40 \mathrm{bc}$ & $706 \mathrm{a}$ \\
T3: estaca continúa con fertilización química & $9,90 \mathrm{cde}$ & $543 \mathrm{~d}$ \\
T4: estaca continúa mezcla fertilización química/abono orgánico & $8,50 \mathrm{e}$ & $561 \mathrm{~cd}$ \\
T5: espaciado con abono orgánico & $10,70 \mathrm{bcd}$ & $650 \mathrm{~b}$ \\
T6: espaciado con fertilización química & $12,10 \mathrm{ab}$ & $594 \mathrm{~cd}$ \\
T7: espaciado mezcla fertilización química/abono orgánico & $10,60 \mathrm{bcd}$ & $593 \mathrm{~cd}$ \\
T8: espaciado con fertilización del agricultor & $13,20 \mathrm{a}$ & $595 \mathrm{c}$ \\
T9: estaca continúa y espaciado sin fertilización & $12,00 \mathrm{ab}$ & $753 \mathrm{a}$ \\
\hline
\end{tabular}

*Promedios con la misma letra no son diferentes estadísticamente $(\mathrm{p}<0,05) / *$ Averages with the same letter are not statistically different $(\mathrm{p}<0.05)$.

\section{Discusión}

Los mayores rendimientos de caña de azúcar para panela o ANC, se obtuvieron en sistema estaca continua con fertilización química, siendo significativamente diferentes con respecto al sistema de estaca continua y espaciado sin fertilización, debido a que la absorción de los nutrientes provenientes de síntesis química ocurre de manera rápida por su proceso de solubilización, en el caso del nitrógeno aplicado a través de la urea y esto de acuerdo a Witte (2011), la enzima ureasa hidroliza la urea descomponiéndola en carbonato de amonio, que posteriormente se descompone en amonio y dióxido de carbono, estando disponible para que lo tome la planta o los microorganismos reduciendo el amonio a nitratos, lo cual es otra forma de absorción por la planta, mientras que en el caso de fertilizantes orgánicos los nutrientes están disponibles en menor contenido, están inmersos en el material particulado, son influenciados por la combinación de factores como las especies vegetales, el tipo de suelo y las condiciones ambientales (Tilak et al., 2005), deben pasar por una primera etapa que la transforma en humus y luego una segunda etapa de evolución lenta, el humus desaparece al convertirse en compuestos minerales, $\mathrm{CO}_{2} \mathrm{y}$ agua, liberándose lentamente en el suelo (García et al., 2007).

El anterior comportamiento se podría atribuir a que, en condiciones de ladera, el sistema de siembra estaca continúa presenta mejor comportamiento frente al espaciado porque el surco a través de la pendiente cierra y forma una barrera que sostiene la humedad y el fertilizante aplicado (Buenaventura y Rangel, 1976; Mafla, 1982), lo que evita las pérdidas por lixiviación y erosión (Chaves, 1999). El rendimiento del tratamiento de siembra espaciado 
con abono orgánico (T5), puede estar relacionado con la población microbiológica presente en el abono orgánico y junto a la humedad retenida por este abono, que mejora procesos aumentando la solubilidad de nutrientes del suelo. Al evaluar el efecto de prácticas orgánicas de nutrición de caña panelera (Gómez, 2016), encontró que los grupos microbianos responden en menor o mayor medida a través del tiempo, de acuerdo al estado de desarrollo del cultivo (Marschner y Rengel, 2007) y las condiciones climáticas que se presentan, por lo tanto, su actividad es específica a las condiciones intrínsecas de la microbiología del suelo (Buscot y Varma, 2005).

Al comparar el rendimiento promedio de los tratamientos donde se usó fertilizante mineral, se encontró que cuando se pasó de fertilización química a mezcla de fuentes, el rendimiento de caña se redujo $15 \%$ y $8 \%$ en siembra en estaca continúa y espaciado, resultado que se puede esperar debido que la mezcla tuvo menos N-P-K de rápida disponibilidad en comparación con la fertilización química. Igualmente, el rendimiento de caña de la mezcla de fuentes fue similar en los dos sistemas de siembra y superó al testigo en $11 \%$, resultados concuerdan con otros estudios (Manrique, 1992; Manrique et al., 2000), donde se han reportado incrementos del $50 \%$ en rendimiento de caña cuando se aplicaron $3 \mathrm{t} \mathrm{ha}^{-1}$ de abono orgánico en mezcla con fuentes minerales de N-P-K, mientras que con dosis entre 10 y $40 \mathrm{~kg} \mathrm{ha}^{-1}$ de abono orgánico, se mejoró la disponibilidad de $\mathrm{K}$ y $\mathrm{P}$ en las dosis más altas y la disponibilidad de $\mathrm{Mg}$ y Na para las dosis más bajas.

Los rendimientos de caña en los tratamientos con solo abono orgánico y en mezcla (abono orgánico/fertilizante mineral), obtuvieron valores mayores a $144 \mathrm{TCH}$ que superaron el promedio de la zona panelera de Nariño estimado en $100 \mathrm{TCH}$, estos resultados evidenciaron los beneficios del abono orgánico, ya que no solo aportó algunos nutrientes sino que también mejoró las condiciones como la estructura del suelo, la retención de humedad (Román et al., 2013), la disminución de fijación del P por el suelo (Rivero y Carracedo, 1999), el aumento de la concentración de bases (Ca, Mg y K) en conjunto con la capacidad de intercambio catiónico (Oorts et al., 2003; Sposito, 2008).

Los mayores rendimientos de panela o ANC se obtuvieron con fertilización química ajustada según el análisis de suelo en sistema de siembra estaca continúa (T3) y en espaciado (T6); con excepción del testigo (T9), los rendimientos de panela o ANC de los tratamientos fueron superiores al promedio de la zona de $12 \mathrm{TPH}$, lo cual demuestra la posibilidad de mejorar la productividad del sistema de producción de caña para panela o ANC con la utilización de abono orgánico y fuentes minerales con base en el diagnóstico de la fertilidad del suelo. Es por esto, que la producción de sacarosa está en función de varios factores entre los que se destaca la variedad, el suelo, prácticas como dosis y épocas de aplicación de nutrientes y madurez (Larrahondo, 1995).

Los rendimientos de panela o ANC obtenidos en los tratamientos donde los niveles de fertilizante químico y abono orgánico se ajustaron con el resultado de análisis de suelo, demostraron y se ajustaron a lo mencionado por Khan et al. (2005) y Mellis et al. (2016), quienes afirmaron que algunos nutrimentos tienen efectos adversos para la calidad y cantidad de sacarosa a cosecha y otros nutrientes mejoran y son claves en la etapa de elaboración de panela o azúcar; excesivo nitrógeno por ejemplo, tiende a disminuir el contenido de sacarosa, los fosfatos solubles son fundamentales para el desarrollo del cultivo y en la recuperación de azúcares. Deficiencias de potasio reducen el contenido de sacarosa y el exceso afecta el proceso de elaboración de panela o ANC y azúcar, ya que el cloruro de potasio cristaliza primero que la sacarosa, de tal manera que las TPH obtenidas en el presente estudio reafirman que los niveles de N-P-K utilizados se constituyen en una referencia importante para mejorar la producción de panela o ANC.

Debido a la situación socioeconómica de los productores de panela o ANC en Colombia, que no les permite realizar un plan de nutrición con fertilizante mineral y debido a que la caña de azúcar es un cultivo altamente extractivo y requiere considerables dosis de macro y micronutrientes, los resultados demostraron que el abono orgánico puede constituirse en una alternativa en el proceso de producción. Pese a que el abono orgánico tiene menor aporte de N-P-K, los resultados pueden relacionarse e interpretarse teniendo en cuenta los requerimientos y absorción de nutrientes por la caña de azúcar reportados (Manrique, et al., 2000; Iqbal e Iqbal, 2014) y por la fertilidad química del suelo (Cuadro 1). 
Los abonos orgánicos compostados, aunque generalmente no son fuente básica de nutrientes en comparación con las fuentes minerales, favorecen el proceso de nutrición porque proporcionan resistencia al estrés (Giri et al., 2003) y ayudan a neutralizar la acidez del suelo (Baquero, 2002; Cui et al., 2002). El rendimiento de panela o ANC con abono orgánico mostró apropiados resultados, solo y en mezcla, en espaciado y estaca continúa el rendimiento fue similar al rendimiento obtenido con fertilización química, lo cual es relevante, especialmente en el caso de la mezcla, debido a que se podría reducir hasta en un $50 \%$ el fertilizante mineral sin afectar el rendimiento, resultado que puede estar relacionado con el efecto lento pero constante del material orgánico a través del largo ciclo de producción de la caña.

Los incrementos en la producción de sacarosa en varias regiones de Colombia, se dieron al utilizar dosis crecientes de N-P-K y abono orgánico, resultados concuerdan con lo reportado por Manrique (2000). En México, Hernández et al. (2008), al evaluar fertilización química y materiales orgánicos, no observaron diferencia significativa en grados brix, sacarosa, azúcares reductores, fibra y humedad, por lo que la calidad de los jugos de la caña de azúcar fue estadísticamente igual a la obtenida con fertilización química. Al evaluar fertilización química y dosis de material orgánico compostado (Arreola-Enriquez et al., 2003), no encontraron diferencias en la calidad del jugo determinada por grados brix, sacarosa, pureza y fibra.

El porcentaje de conversión de caña a panela o rendimiento a panela o ANC es de gran importancia técnica y económica, debido a que está relacionado con factores como costos de mano de obra, combustible, evaporación, transporte, entre otros; a diferencia del rendimiento de caña y panela o ANC, el porcentaje de conversión disminuyó en los tratamientos con menor aporte de N-P-K (Cuadro 5), debido a que la caña demanda altas dosis de nutrientes, lo cual está asociado a la concentración de azúcares.

En general, el contenido de azúcares reductores obtenido en los tratamientos fue alto según los rangos estipulados en algunas normas técnicas sobre calidad de la panela (779 de 2006) y con lo reportado en trabajos (Keshavaiah, 2011; Ramírez et al., 2014) donde se encontraron valores máximos de $6 \%$; por tanto, la calidad del azúcar crudo y de la panela o ANC, dependen, en parte, de los azúcares reductores que se incrementan por varias causas como el manejo agronómico del cultivo, manejo de la caña en cosecha y el deterioro después del corte (Clarke et al., 1986; Larrahondo, 1995; Mujica et al., 2007). Este deterioro empieza inmediatamente después del corte o cosecha, siendo mayor a medida que aumenta el tiempo de permanencia en los patios del molino o en el campo; la tasa de deterioro depende de las condiciones ambientales, de la variedad y del sistema de manejo que incluye infecciones bacterianas (Larrahondo, 1995; Zossi et al., 2010).

El comportamiento del contenido de azúcares reductores, no mostró una tendencia clara respecto a los tratamientos, puede estar relacionado con el manejo de la caña desde la cosecha en la zona de estudio de Nariño hasta el procesamiento que se realizó en AGROSAVIA en el centro de investigación Tibaitata sede CIMPA, en Barbosa (Santander) que incluyó el envió de las muestras de caña en transporte terrestre a más de $1000 \mathrm{~km}$ de distancia y con el tiempo transcurrido de cosecha a proceso de aproximadamente seis días. Adicionalmente, las coloraciones presentadas en las panelas fueron oscuras, causadas por la alta concentración de azucares reductores, lo cual concuerda con Larrahondo (1983) y Durán (2010), quienes mencionan que un contenido aceptable en panela o ANC no debe superar el $10 \%$, debido a que presenta problemas de calidad, en cuanto a su consistencia y su vida útil.

Respecto al contenido de fosfatos en panela o ANC, aunque hubo diferencia entre tratamientos, el comportamiento del sistema de siembra en estaca continúa con abono orgánico (T2) y estaca continúa y espaciado sin fertilización (T9), se explica debido a que a medida que aumenta el contenido de fosfatos la producción de toneladas de panela o ANC (TPH) es menor, porque la planta exige mayor requerimiento energético (Marschner, 1995), haciendo que los iones de fósforo se distribuyan a la síntesis de moléculas como adenosín trifosfato (ATP) y nicotinamida adenina dinucleótido fosfato (NADP).

En términos generales, el contenido de fosfatos en panela o ANC de los tratamientos, estuvo entre 514 y 753 ppm, fue similar a lo reportado por Mascietti (2014) y Prada-Forero et al. (2015) y puede estar relacionado con el 
efecto de la materia orgánica que reduce la fijación de fósforo (Baquero, 2002) en Andisoles y facilita su absorción; es importante mencionar que los valores están dentro de los rangos aceptables mencionados en la norma sobre higiene y calidad de la panela (779 de 2006).

\section{Conclusiones}

La fertilización química basada en requerimientos nutricionales y fertilidad del suelo presentó los mayores rendimientos de panela o ANC por hectárea, de la misma manera que la fertilización con abono orgánico. Por lo que tecnificar los planes de fertilización a partir de análisis de suelos debe ser un factor que se debe validar dentro del modelo productivo de caña de azúcar para panela o ANC del departamento de Nariño en Colombia.

El uso de planes de fertilización con abonos orgánicos solos y en combinación con fertilizantes de síntesis química demostró su potencial en términos de producción de toneladas de caña por hectárea (TCH) y toneladas de panela o ANC por hectárea (TPH), frente al testigo absoluto y al modelo de fertilización del agricultor; sin embargo, su mayor impacto fue la disminución de fertilizantes de síntesis química, con el respectivo aumento en los beneficios económicos y los beneficios futuros en la fertilidad natural del suelo.

\section{Agradecimientos}

A la Corporación Colombiana de Investigación Agropecuaria (AGROSAVIA) del centro de investigación (C.I.) Obonuco (Pasto, Nariño) y C.I. Tibaitatá sede CIMPA (Barbosa, Santander), por haber formulado y ejecutado la investigación para promover el mejoramiento de la productividad a través de prácticas de manejo en la zona panelera de Nariño (Colombia).

\section{Conflictos de intereses}

El manuscrito fue preparado y revisado con la participación de todos los autores, quienes declaramos que no existe conflicto de intereses que ponga en riesgo la validez de los resultados presentados.

\section{Financiación}

Este estudio fue financiado con fondos del Ministerio de Agricultura y Desarrollo Rural (MADR) y el Instituto Interamericano de Cooperación para la Agricultura (IICA).

\section{Literatura citada}

Aguiar, S.B. 2001. Bases técnicas para el establecimiento y manejo del cultivo de caña en el departamento de Casanare. Boletín Técnico No. 24. Corporación Colombiana de Investigación Agropecuaria (CORPOICA), Yopal, COL.

Agronet (Red de Información y Comunicación del Sector Agropecuario). 2016. Estadísticas agrícolas. Agronet, COL. http:// www.agronet.gov.co/estadistica/Paginas/default.aspx (consultado 2 nov. 2018).

Arreola-Enriquez, J., D.J. Palma-Lopez, S. Salgado-Garcia, W. Camacho-Chiu, J.J. Obrador-Olan, J.F. Juarez-Lopez, y L. Pastrana-Aponte. 2003. Evaluación de abono órgano-mineral de cachaza en la producción y calidad de la caña de azúcar. Terra Latinoam. 22:351-357. 
Baquero, J.E. 2002. Estudio de la interacción suelo planta resultante de la adición de materiales orgánicos en cultivares de maíz en un oxisol de la Orinoquia Colombiana. Tesis MSc., Universidad Nacional de Colombia, Bogotá, COL.

Bonilla, I. 2008. Introducción a la nutrición mineral de las plantas. Los elementos minerales. En: J. Azcón, y M. Talón, editores, Fundamentos de fisiología vegetal. McGraw-Hill, Madrid, ESP. p. 103-122.

Borges, L.D.A., B.E. Madari, W.M. Leandro, P.M. Fernandes, E.A. da-Silva, and M.A.S. da-Silva. 2015. Nutritional state and productivity of organic sugarcane in Goias, Brasil. J. Agron. 14:6-14. doi:10.3923/ja.2015.6.14

Buenaventura, O., y J. Rangel. 1976. Estudios sobre métodos, distancias y densidades de siembra en caña para panela. Reporte de investigación \#52. Instituto Colombiano Agropecuario (ICA), Bogotá, COL.

Buscot, F., and A. Varma (ed). 2005. Microorganisms in soils: Roles in genesis and functions. Springer, DNK.

Campuzano, L., y A. Bolaños. 2003. Difusión del manejo de control biológico en la caña panelera en cinco municipios de la zona sur occidente del departamento de Nariño, San Juan de Pasto. CORPOICA, Nariño, COL.

Chaves, M. 1999. El nitrógeno, fósforo y potasio en la caña de azúcar. Liga Agrícola Industrial de la caña de azúcar, San José, CRI.

Chi, L., J. Mendoza-Vega, E. Huerta, and J.D. Álvarez-Solís. 2017. Effect of long-term sugarcane Saccharum spp. cultivation on chemical and physical properties of soils in Belize. Commun. Soil Sci. Plant Anal. 48:741-755. doi:10.1080/001036 24.2016.1254794

Clarke, M., R. Blanco, and M. Godshall. 1986. Colorant in raw sugars. In: ISSCT, editor, Proceedings of International Society Sugar Cane Technologist. ISSCT, Georgetown, MA, USA. p. 670-682.

Cui, J.Y., Y.L. Li, and Y.Z. Su. 2002. Experiment of using fermented waste residue from alcohol production to improve sandy soil. J. Desert Res. 4:368-371.

DANE (Departamento Administrativo Nacional de Estadística). 2017. Insumos y factores asociados a la producción agropecuaria. Boletín mensual \#57. DANE, Bogotá, COL.

Dotaniya, M.L., S.C. Datta, D.R. Biswas, C.K. Dotaniya, B.L. Meena, S. Rajendiran, K.L. Regar, and M. Lata. 2016. Use of sugarcane industrial by-products for improving sugarcane productivity and soil health. Int. J. Recycl. Org. Waste Agric. 5:185. doi:10.1007/s40093-016-0132-8

Durán, N. 2010. Reingeniería panelera. Editorial Produmedios, Bogotá, COL.

Eaton, A.D., APHA (American Public Health Association), AWWA (American Water Works Association), and WEF (Water Environment Federation). 2005. Standard methods for the examination of water and wastewater. 21th ed. Method 4500PA, C y E. APHA, AWWA, and WEF, WA, USA.

Estupiñán, R.M., O. Insuasty, C.J. Mora, G. Rodríguez, R. Blanco, L.A. Mejía, J. Libardo, y G. Sandoval. 2000. Manual de caña de azúcar para la producción de panela. CORPOICA, Bucaramanga, COL.

García, H.R., L.C. Albarracin, A. Toscano, N.J. Santana, y O. Insuasty. 2007. Guía tecnológica para el manejo integral del sistema productivo de la caña panelera. CORPOICA, Bogota, COL. http://hdl.handle.net/11348/4009 (consultado 15 nov. 2018).

García, J.C., M.S. Scarpari, M.G. Landell, and A.A. Cologna. 2010. Use of organomineral fertilisers on sugarcane productivity in a typic haplustox soil. In: ISSCT, editor, Proceedings of International Society Sugar Cane Technologist. ISSCT, Georgetown, MA, USA. http://www.issct.org/pdf/proceedings/2010/2010\%20Garcia,\%20USE\%20OF\%20ORGANOMINERAL\%20 FERTILISERS \%20ON\%20SUGARCANE\%20PRODUCTIVITY\%20IN\%20A\%20TYPIC\%20HAPLUSTOX\% 20 SOIL.pdf (consultado 20 nov. 2018) 
Giri, B., R. Kapoor, and G. Mukerjik. 2003. Influence of arbuscular mycorrhizal fungi and salinity on growth, biomass and mineral nutrition of Acacia auriculiformis. Biol. Fertil. Soils 38:170-175. doi:10.1007/s00374-003-0636-Z

Gómez, R.M. 2016. Evaluación del efecto de prácticas orgánicas de nutrición de caña panelera (Saccharum officinarum L.) en el municipio de Nocaima - Cundinamarca. Tesis MSc., Universidad Nacional. Bogotá, COL. http://bdigital.unal.edu. co/52459/1/Tesis\%20Ruth\%20Milena\%20G\%C3\%B3mez\%20Vargas.pdf (consultado 22 may. 2019).

Gopalasundaram, P., A. Bhaskaran, and P. Rakkiyappan. 2012. Integrated nutrient management in sugarcane. Sugar Tech 14:320. doi:10.1007/s12355-011-0097-x

Guaman, F., E. Guaman, y H. Villavicencio. 2009. Diseño, simulación y emulación de una planta productora de panela. Escuela Superior Politécnica del Litoral (ESPOL), ECU. http://www.dspace.espol.edu.ec/bitstream/123456789/1045/1/1929.pdf (consultado 20 feb. 2019).

Gutiérrez-Mosquera, L.F, S. Arias-Giraldo, y A.M. Ceballos-Peñaloza. 2018. Actualidad del sistema productivo tradicional de panela en Colombia: análisis de mejoras y alternativas tecnológicas. Ingeniería y Competitividad 20(1):107-123. doi:10.25100/iyc.v20i1.6190

Hernández, G.I., S. Salgado, D.J. Palma, L.C. Lagunes-Espinoza, M. Castelán, y O. Ruiz. 2008. Vinaza y composta de cachaza como fuente de nutrientes en caña de azúcar en un gleysol mólico de Chiapas. Interciencia 33:860-855.

IGAC (Instituto Geográfico Agustín Codazzi). 2004. Estudio general de suelos y zonificación de tierras, Departamento de Nariño. Geografía Oficial de Colombia, Bogotá, Colombia.

Iqbal, M.A., and A. Iqbal. 2014. Sugarcane production, economics and industry in Pakistan. American-Eurasian J. Agric. Environ. Sci. 14:1470-1477. doi:10.5829/idosi.aejaes.2014.14.12.12479

Keshavaiah, K. 2011. Effect of organic and integrated nutrient management practices on growth, yield and quality of sugarcane and jaggery in cauvery command area. Ph.D. Diss., Dharwad University of Agricultural Sciences, Karnataka, IND.

Khan, I.A., A. Khatri, M.A. Siddiqui, N.A. Dahar, G:S. Nizamani, and S.Raza. 2005. Effect of NPK fertilizer on the growth of sugarcane clone AEC 86347 developed at NIA Tando Jam Pakistan. Pak. J. Bot. 37:355-360.

Lane, J.H., and L. Eynon. 1923. Volumetric determination of reducing sugars by means of Fehling's solution, with methylene blue as internal indicator. Int. Sug. J. 25:143-149.

Larrahondo, J.E. 1983. El deterioro de la caña de azúcar después del corte bajo los sistemas de cosecha mecánica y manual. En: Sociedad Colombiana de Técnicos de la Caña de Azúcar (TECNICAÑA), editor, Seminario cosecha mecanizada de la caña de azúcar. CENICAÑA, Cali, COL. p. 77-85.

Larrahondo, J.E. 1995. Calidad de la caña de azúcar. En: C. Cassalett et al., editores, El cultivo de la caña de azúcar en la zona azucarera de Colombia. CENICAÑA, Cali, COL. p. 337-354.

Larrahondo, J., y F. Villegas. 1995. Control y características de maduración. CENICAÑA, Cali, COL.

Latief, A.S., R. Syarief, B. Pramudya, and M. Muhadiono. 2010. Productivity of sugarcane plants of ratooning with fertilizing treatment. Nusantara Biosci. 2(1):43-47. doi:10.13057/nusbiosci/n020107

Loneragan, J.F. 1997. Plant nutrition in the $20^{\text {th }}$ and perspectives for the $21^{\text {st }}$ century. Plant Soil 196:63-174. doi:10.1023/A:1004208621263

Luna, E. 1992. Evaluación del proceso de producción de panela en el departamento de Nariño. Rev. Cienc. Agric. 11:1-8.

Mafla, A. 1982. Efecto de diferentes distancias y densidades de siembra en la producción de caña para panela. Rev. ICA 17(4):141-148. 
Maldonado, F.G., M.N. Santana, y J.D. Jiménez. 2006. Prácticas sostenibles en el sistema de caña panelera. Publicación técnica 47. Corporación Colombiana de Investigación Agropecuaria (CORPOICA), Bogotá, COL.

Manrique, R. 1992. Nutrición y fertilización de la caña de azúcar para panela. En: Colombiana de Investigación Agropecuaria (CORPOICA), editores, Manual de caña para panela Convenio ICA-Holanda de investigación y divulgación para el mejoramiento de la industria panelera en Colombia. CORPOICA, COL. p. 93.

Manrique, R., J. Durán, M.L. Murcia, O. Insuasty, A.M. Bayona, E. Sanabria, y M.A. Alfonso. 2010. Invespanela. Módulo 1: Establecimiento de cultivo y levante de socas. Lección 1. Alternativas para la adecuación del terreno. Universidad de Pamplona, Cucuta, COL. http://www.unipamplona.edu.co/unipamplona/portalIG/home_4/mod_virtuales/modulo1/ leccin_1.html (consultado 22 may. 2019).

Manrique, R., O. Insuasty, C.J. Mora, G. Rodríguez, R. Blanco, J.L. Pinto, L. Mejía, y G. Sandoval. 2000. Manual de caña de azúcar para la producción de panela. Corporación Colombiana de Investigación Agropecuaria (CORPOICA), y Servicio Nacional de Aprendizaje (SENA), Bogotá, COL.

Marschner, H. 1995. Mineral nutrition in higher plants. $2^{\text {nd }}$ ed. American Press, London, GBR.

Marschner, P., and Z. Rengel. 2007. Soil biology. Nutrient cycling in terrestrial ecosystems. Springer, Berlin, DEU.

Martínez, D. 2013. Agenda prospectiva de investigación de la cadena productiva de la panela y su agroindustria. Tecnura 17(36):72-86.

Mascietti, M. 2014. PANELA: Propiedades, información y aceptación. Tesis Lic., Universidad Fraternidad de Agrupaciones Santo Tomás de Aquino, Buenos Aires, ARG.

Mendez, H. 1981. Fertilización de la caña de azúcar (Saccharum officinarum L) para panela en suelos de ladera del departamento de Nariño. Instituto Colombiano Agropecuario (ICA), Bogotá, COL.

Mellis, E.V., J.A. Quaggio, G.R.G. Becari, L.A.J. Teixeira, H. Cantarella, and F.L.F. Dias. 2016. Effect of micronutrients soil supplementation on sugarcane in different production environments: Cane plant cycle. Soil Fertil. Crop Nutr. 108:20602070. doi:10.2134/agronj2015.0563

Mujica, M., M. Guerra, y N. Soto. 2007. Efecto de la variedad, lavado de la caña y temperatura de punteo sobre la calidad de la panela granulada. Interciencia 33:598-603.

Muñoz, R. 1978. Características generales de suelos y fertilización de la caña para panela en la región andina colombiana. Instituto Colombiano Agropecuario (ICA), Bogotá, COL.

Muñoz, A.F. 2012. Evaluación de la aplicación de compost en el cultivo de la caña de azúcar. En: Sociedad Colombiana de la Ciencia del Suelo (SCCS), editores, Memorias XI Congreso Colombiano de la Ciencia del Suelo. Sociedad Colombiana de la Ciencia del Suelo (SCCS), Riohacha, COL. p. 128.

Oorts, K., B. Vanlauwe, y R. Merckx. 2003. Cation exchange capacities of soil organic matter fractions in a Ferric Lixisol with different organic matter inputs. Agric. Ecosyst. Environ. 100:161-171. doi:10.1016/S0167-8809(03)00190-7

Osorio, G. 2007. Manual técnico buenas prácticas agrícolas -BPA- y buenas prácticas de manufactura -BPM-en la producción de caña y panela. CTP Print Ltda., Medellín, COL.

Palled, Y.B. 2011. Effect of organic and integrated nutrient management practices on growth, yield and quality of sugarcane and jaggery in cauvery command area. Ph.D. Diss., University of Agricultural Sciences, Bangalore, IND.

Prada-Forero, L.E., A. Chaves-Guerrero, y H.R. García-Bernal. 2015. Efecto de la presión de evaporación y la variedad de caña sobre la calidad de la miel y la panela. Cienc. Tecnol. Agropecu. 16:153-165. 
Quintero, R. 1995. Fertilización y nutrición. En: D. Casalett et al., editores, El cultivo de la caña. CENICAÑA, Cali, COL. p. 153-157.

Ramírez, J., O. Insuasty, y C.A. Viveros. 2014. Comportamiento agroindustrial de diez variedades de caña de azúcar para producción de panela en Santander, Colombia. Cienc. Tecnol. Agropecu. 15(2):183-195.

Rangel, M.C., R. Manrique, O. Insuasty, B. Volverás, J. Gómez, H. López, y G. Rodríguez. 2006. Línea base sistema productivo caña de azúcar para panela. Documento técnico. CORPOICA, Barbosa, COL.

Requier-Desjardins, D., and G. Rodríguez. 2004. Environmental impact of panela food-processing industry: sustainable agriculture and local agri-food production systems. Int. J. Sustain. Dev. 7:237-256. doi:10.1504/IJSD.2004.005956

Rivero, C., y C. Carracedo. 1999. Efecto del uso de gallinaza sobre algunos parámetros de fertilidad química de dos suelos de pH contrastante. Rev. Fac. Agron. 25:83-93.

Rodríguez, G. 2000. La panela en Colombia frente al nuevo milenio: Un análisis de la cadena agroindustrial. $2^{\text {da }}$ ed. En: Fedepanela-SENA, editores, Manual de caña para la producción de panela. Corporación Colombiana de Investigación Agropecuaria (CORPOICA), Bucaramanga, COL. p. 10-24.

Román, P., M. Martínez, y A. Pantoja. 2013. Manual de compostaje del agricultor. Experiencias en América Latina. FAO, Santiago, CHL. http://www.fao.org/3/a-i3388s.pdf (consultado 22 may. 2019).

Shivanaikar, M. 2012. Organic and inorganic cultivation of sugarcane and jaggery preparation in Bagalkot district: An economic analysis. Thesis MSc., University of Agricultural Sciences, Dharwad, IND.

Sposito, G. 2008. The chemistry of soils. $2^{\text {nd }}$ ed. Oxford University Press, NY, USA.

Tilak, K.V.B.R., N. Ranganayaki, K.K. Pal, R. De, A.K. Saxena, C. Shekhar, S. Mittal, A.K. Tripathi, and B.N. Johri. 2005. Diversity of plant growth and soil health supporting bacteria. Current Sci. 89:136-150.

Torres, R., L. Soto, O. Collazos, y J. Ante. 1998. Manejo agronómico de la caña panelera en la meseta de Popayán, Cauca. Cartilla No 4. CORPOICA, Cauca, COL.

Volverás, B., y E. Amézquita. 2009. Estabilidad estructural del suelo bajo diferentes sistemas y tiempos de uso en laderas andinas de Nariño, Colombia. Acta Agron. 58(1):35-39.

Witte, C. 2011. Urea metabolism in plants. Plant Sci.180:431-438. doi:10.1016/j.plantsci.2010.11.010

Zossi, S., G. Cárdenas, N. Sorol, y M. Sastre. 2010. Influencia de compuestos azúcares y no azúcares en la calidad industrial de caña de azúcar en Tucumán: Parte 1: caña limpia y despuntada. Rev. Ind. Agric. Tucumán 87(1):15-27. 